\title{
Modeling the PVTx Behavior of the N-Methylpyrrolidinone/Water Mixed Solvent
}

\author{
Begoña García, Santiago Aparicio, Rafael Alcalde, María J. Dávila, and José M. Leal* \\ Universidad de Burgos, Departamento de Química, 09001 Burgos, Spain
}

*Corresponding Author: Phone: +34 947258 819; Fax: +34 947258 831; E-Mail:
jmleal@ubu.es

Supporting Information 
Table I. Experimental densities, $\rho$, for the $x N M P+(1-x)$ water binary solvents as a function of pressure and temperature

\begin{tabular}{|c|c|c|c|c|c|c|c|c|c|}
\hline \multirow[b]{2}{*}{$\begin{array}{c}P \\
(M P a)\end{array}$} & \multicolumn{9}{|c|}{$T(K)$} \\
\hline & 278.15 & 288.15 & 298.15 & 308.15 & 318.15 & 328.15 & 338.15 & 348.15 & 358.15 \\
\hline & \multicolumn{9}{|c|}{$x=0.0981$} \\
\hline 0.10 & 1.0381 & 1.0325 & 1.0262 & 1.0197 & 1.0124 & 1.0052 & 0.9977 & 0.9893 & 0.9810 \\
\hline 1.00 & 1.0384 & 1.0328 & 1.0265 & 1.0200 & 1.0128 & 1.0056 & 0.9980 & 0.9898 & 0.9815 \\
\hline 5.00 & 1.0398 & 1.0343 & 1.0280 & 1.0216 & 1.0144 & 1.0073 & 0.9998 & 0.9916 & 0.9834 \\
\hline 10.00 & 1.0416 & 1.0361 & 1.0299 & 1.0235 & 1.0164 & 1.0094 & 1.0020 & 0.9939 & 0.9858 \\
\hline 15.00 & 1.0433 & 1.0379 & 1.0317 & 1.0254 & 1.0184 & 1.0114 & 1.0041 & 0.9961 & 0.9877 \\
\hline 20.00 & 1.0450 & 1.0396 & 1.0335 & 1.0273 & 1.0203 & 1.0134 & 1.0062 & 0.9983 & 0.9904 \\
\hline 25.00 & 1.0467 & 1.0413 & 1.0352 & 1.0291 & 1.0222 & 1.0154 & 1.0083 & 1.0004 & 0.9926 \\
\hline 30.00 & 1.0484 & 1.0431 & 1.0370 & 1.0309 & 1.0241 & 1.0174 & 1.0103 & 1.0025 & 0.9948 \\
\hline 35.00 & 1.0501 & 1.0448 & 1.0388 & 1.0327 & 1.0259 & 1.0193 & 1.0122 & 1.0046 & 0.9970 \\
\hline 40.00 & 1.0517 & 1.0465 & 1.0405 & 1.0344 & 1.0278 & 1.0212 & 1.0142 & 1.0067 & 0.9991 \\
\hline 45.00 & 1.0533 & 1.0481 & 1.0422 & 1.0362 & 1.0296 & 1.0231 & 1.0162 & 1.0087 & 1.0012 \\
\hline 50.00 & 1.0550 & 1.0498 & 1.0439 & 1.0380 & 1.0314 & 1.0249 & 1.0181 & 1.0107 & 1.0033 \\
\hline 55.00 & 1.0566 & 1.0514 & 1.0456 & 1.0396 & 1.0332 & 1.0267 & 1.0200 & 1.0126 & 1.0053 \\
\hline \multirow[t]{2}{*}{60.00} & 1.0580 & 1.0529 & 1.0471 & 1.0412 & 1.0347 & 1.0284 & 1.0217 & 1.0145 & 1.0072 \\
\hline & \multicolumn{9}{|c|}{$x=0.2002$} \\
\hline 0.10 & 1.0592 & 1.0511 & 1.0426 & 1.0340 & 1.0251 & 1.0162 & 1.0069 & 0.9975 & 0.9878 \\
\hline 1.00 & 1.0595 & 1.0514 & 1.0429 & 1.0343 & 1.0255 & 1.0167 & 1.0073 & 0.9979 & 0.9883 \\
\hline 5.00 & 1.0609 & 1.0530 & 1.0445 & 1.0359 & 1.0272 & 1.0185 & 1.0092 & 1.0000 & 0.9904 \\
\hline 10.00 & 1.0627 & 1.0549 & 1.0464 & 1.0379 & 1.0294 & 1.0206 & 1.0115 & 1.0024 & 0.9930 \\
\hline 15.00 & 1.0645 & 1.0566 & 1.0483 & 1.0399 & 1.0314 & 1.0228 & 1.0138 & 1.0047 & 0.9955 \\
\hline 20.00 & 1.0662 & 1.0584 & 1.0502 & 1.0419 & 1.0334 & 1.0249 & 1.0160 & 1.0071 & 0.9979 \\
\hline 25.00 & 1.0679 & 1.0601 & 1.0520 & 1.0438 & 1.0354 & 1.0270 & 1.0182 & 1.0094 & 1.0003 \\
\hline 30.00 & 1.0695 & 1.0619 & 1.0538 & 1.0456 & 1.0374 & 1.0290 & 1.0203 & 1.0116 & 1.0027 \\
\hline 35.00 & 1.0712 & 1.0636 & 1.0555 & 1.0474 & 1.0393 & 1.0311 & 1.0224 & 1.0138 & 1.0050 \\
\hline 40.00 & 1.0728 & 1.0653 & 1.0573 & 1.0492 & 1.0412 & 1.0330 & 1.0245 & 1.0160 & 1.0073 \\
\hline 45.00 & 1.0744 & 1.0669 & 1.0590 & 1.0510 & 1.0430 & 1.0350 & 1.0266 & 1.0181 & 1.0096 \\
\hline 50.00 & 1.0760 & 1.0686 & 1.0607 & 1.0528 & 1.0449 & 1.0369 & 1.0286 & 1.0202 & 1.0117 \\
\hline 55.00 & 1.0775 & 1.0702 & 1.0624 & 1.0545 & 1.0467 & 1.0388 & 1.0306 & 1.0223 & 1.0139 \\
\hline \multirow[t]{2}{*}{60.00} & 1.0791 & 1.0717 & 1.0639 & 1.0562 & 1.0483 & 1.0405 & 1.0323 & 1.0243 & 1.0159 \\
\hline & \multicolumn{9}{|c|}{$x=0.3000$} \\
\hline 0.10 & 1.0652 & 1.0564 & 1.0473 & 1.0379 & 1.0285 & 1.0191 & 1.0094 & 0.9992 & 0.9892 \\
\hline 1.00 & 1.0655 & 1.0567 & 1.0476 & 1.0383 & 1.0289 & 1.0195 & 1.0098 & 0.9997 & 0.9897 \\
\hline 5.00 & 1.0670 & 1.0583 & 1.0493 & 1.0401 & 1.0307 & 1.0214 & 1.0119 & 1.0019 & 0.9920 \\
\hline 10.00 & 1.0689 & 1.0603 & 1.0514 & 1.0422 & 1.0329 & 1.0238 & 1.0143 & 1.0045 & 0.9948 \\
\hline 15.00 & 1.0707 & 1.0621 & 1.0533 & 1.0443 & 1.0352 & 1.0260 & 1.0167 & 1.0070 & 0.9975 \\
\hline 20.00 & 1.0725 & 1.0640 & 1.0553 & 1.0463 & 1.0373 & 1.0283 & 1.0191 & 1.0095 & 1.0001 \\
\hline 25.00 & 1.0743 & 1.0658 & 1.0572 & 1.0484 & 1.0394 & 1.0305 & 1.0214 & 1.0120 & 1.0027 \\
\hline 30.00 & 1.0760 & 1.0677 & 1.0591 & 1.0503 & 1.0415 & 1.0327 & 1.0237 & 1.0143 & 1.0052 \\
\hline 35.00 & 1.0778 & 1.0694 & 1.0609 & 1.0522 & 1.0435 & 1.0348 & 1.0259 & 1.0167 & 1.0076 \\
\hline 40.00 & 1.0795 & 1.0712 & 1.0628 & 1.0541 & 1.0455 & 1.0369 & 1.0281 & 1.0190 & 1.0100 \\
\hline 45.00 & 1.0811 & 1.0729 & 1.0646 & 1.0560 & 1.0475 & 1.0389 & 1.0303 & 1.0212 & 1.0124 \\
\hline 50.00 & 1.0828 & 1.0746 & 1.0664 & 1.0579 & 1.0494 & 1.0410 & 1.0324 & 1.0234 & 1.0147 \\
\hline 55.00 & 1.0844 & 1.0763 & 1.0682 & 1.0597 & 1.0513 & 1.0429 & 1.0345 & 1.0256 & 1.0170 \\
\hline 60.00 & 1.0859 & 1.0780 & 1.0698 & 1.0614 & 1.0530 & 1.0449 & 1.0364 & 1.0276 & 1.0192 \\
\hline
\end{tabular}


Table I. Continued

\begin{tabular}{|c|c|c|c|c|c|c|c|c|c|}
\hline \multirow{2}{*}{$\begin{array}{c}P \\
(M P a)\end{array}$} & \multicolumn{9}{|c|}{$T(K)$} \\
\hline & 278.15 & 288.15 & 298.15 & 308.15 & 318.15 & 328.15 & 338.15 & 348.15 & 358.15 \\
\hline & \multicolumn{9}{|c|}{$x=0.4009$} \\
\hline 0.10 & 1.0644 & 1.0556 & 1.0464 & 1.0370 & 1.0273 & 1.0176 & 1.0080 & 0.9980 & 0.9879 \\
\hline 1.00 & 1.0647 & 1.0559 & 1.0467 & 1.0374 & 1.0278 & 1.0181 & 1.0085 & 0.9985 & 0.9885 \\
\hline 5.00 & 1.0664 & 1.0576 & 1.0485 & 1.0393 & 1.0297 & 1.0201 & 1.0107 & 1.0008 & 0.9909 \\
\hline 10.00 & 1.0683 & 1.0597 & 1.0506 & 1.0415 & 1.0321 & 1.0226 & 1.0133 & 1.0035 & 0.9938 \\
\hline 15.00 & 1.0703 & 1.0617 & 1.0527 & 1.0437 & 1.0344 & 1.0250 & 1.0159 & 1.0062 & 0.9966 \\
\hline 20.00 & 1.0722 & 1.0637 & 1.0548 & 1.0459 & 1.0366 & 1.0274 & 1.0184 & 1.0088 & 0.9994 \\
\hline 25.00 & 1.0740 & 1.0656 & 1.0568 & 1.0480 & 1.0389 & 1.0297 & 1.0208 & 1.0114 & 1.0021 \\
\hline 30.00 & 1.0759 & 1.0675 & 1.0588 & 1.0500 & 1.0410 & 1.0320 & 1.0232 & 1.0139 & 1.0047 \\
\hline 35.00 & 1.0777 & 1.0694 & 1.0608 & 1.0521 & 1.0432 & 1.0342 & 1.0255 & 1.0164 & 1.0073 \\
\hline 40.00 & 1.0795 & 1.0712 & 1.0627 & 1.0541 & 1.0453 & 1.0364 & 1.0278 & 1.0188 & 1.0098 \\
\hline 45.00 & 1.0813 & 1.0731 & 1.0646 & 1.0560 & 1.0473 & 1.0386 & 1.0301 & 1.0211 & 1.0123 \\
\hline 50.00 & 1.0829 & 1.0748 & 1.0665 & 1.0580 & 1.0493 & 1.0407 & 1.0322 & 1.0234 & 1.0147 \\
\hline 55.00 & 1.0847 & 1.0766 & 1.0683 & 1.0599 & 1.0513 & 1.0427 & 1.0344 & 1.0257 & 1.0171 \\
\hline \multirow[t]{2}{*}{60.00} & 1.0862 & 1.0782 & 1.0701 & 1.0617 & 1.0531 & 1.0447 & 1.0364 & 1.0279 & 1.0193 \\
\hline & \multicolumn{9}{|c|}{$x=0.5023$} \\
\hline 0.10 & 1.0613 & 1.0525 & 1.0432 & 1.0341 & 1.0247 & 1.0152 & 1.0056 & 0.9956 & 0.9858 \\
\hline 1.00 & 1.0617 & 1.0529 & 1.0436 & 1.0345 & 1.0252 & 1.0156 & 1.0061 & 0.9961 & 0.9864 \\
\hline 5.00 & 1.0634 & 1.0547 & 1.0454 & 1.0364 & 1.0272 & 1.0178 & 1.0083 & 0.9985 & 0.9888 \\
\hline 10.00 & 1.0654 & 1.0568 & 1.0477 & 1.0388 & 1.0297 & 1.0204 & 1.0111 & 1.0014 & 0.9919 \\
\hline 15.00 & 1.0675 & 1.0589 & 1.0499 & 1.0411 & 1.0322 & 1.0229 & 1.0138 & 1.0042 & 0.9947 \\
\hline 20.00 & 1.0695 & 1.0610 & 1.0521 & 1.0433 & 1.0345 & 1.0254 & 1.0164 & 1.0070 & 0.9978 \\
\hline 25.00 & 1.0714 & 1.0630 & 1.0542 & 1.0455 & 1.0368 & 1.0278 & 1.0189 & 1.0097 & 1.0006 \\
\hline 30.00 & 1.0733 & 1.0650 & 1.0563 & 1.0477 & 1.0391 & 1.0302 & 1.0214 & 1.0123 & 1.0034 \\
\hline 35.00 & 1.0752 & 1.0670 & 1.0583 & 1.0498 & 1.0413 & 1.0325 & 1.0238 & 1.0148 & 1.0060 \\
\hline 40.00 & 1.0771 & 1.0689 & 1.0603 & 1.0519 & 1.0435 & 1.0348 & 1.0262 & 1.0173 & 1.0086 \\
\hline 45.00 & 1.0789 & 1.0709 & 1.0623 & 1.0539 & 1.0456 & 1.0370 & 1.0285 & 1.0197 & 1.0112 \\
\hline 50.00 & 1.0807 & 1.0727 & 1.0642 & 1.0560 & 1.0477 & 1.0392 & 1.0308 & 1.0221 & 1.0137 \\
\hline 55.00 & 1.0825 & 1.0745 & 1.0662 & 1.0579 & 1.0497 & 1.0413 & 1.0331 & 1.0245 & 1.0161 \\
\hline \multirow[t]{2}{*}{60.00} & 1.0842 & 1.0762 & 1.0679 & 1.0598 & 1.0518 & 1.0432 & 1.0352 & 1.0267 & 1.0185 \\
\hline & \multicolumn{9}{|c|}{$x=0.6013$} \\
\hline 0.10 & 1.0574 & 1.0488 & 1.0397 & 1.0307 & 1.0213 & 1.0120 & 1.0026 & 0.9929 & 0.9830 \\
\hline 1.00 & 1.0578 & 1.0492 & 1.0401 & 1.0311 & 1.0217 & 1.0125 & 1.0032 & 0.9934 & 0.9836 \\
\hline 5.00 & 1.0596 & 1.0510 & 1.0421 & 1.0332 & 1.0238 & 1.0148 & 1.0055 & 0.9959 & 0.9863 \\
\hline 10.00 & 1.0617 & 1.0532 & 1.0444 & 1.0356 & 1.0264 & 1.0175 & 1.0084 & 0.9989 & 0.9894 \\
\hline 15.00 & 1.0638 & 1.0555 & 1.0467 & 1.0380 & 1.0289 & 1.0201 & 1.0112 & 1.0018 & 0.9925 \\
\hline 20.00 & 1.0659 & 1.0576 & 1.0490 & 1.0404 & 1.0314 & 1.0227 & 1.0138 & 1.0047 & 0.9955 \\
\hline 25.00 & 1.0679 & 1.0597 & 1.0512 & 1.0426 & 1.0338 & 1.0252 & 1.0165 & 1.0074 & 0.9984 \\
\hline 30.00 & 1.0699 & 1.0618 & 1.0533 & 1.0448 & 1.0361 & 1.0276 & 1.0191 & 1.0101 & 1.0012 \\
\hline 35.00 & 1.0719 & 1.0638 & 1.0554 & 1.0470 & 1.0384 & 1.0300 & 1.0216 & 1.0127 & 1.0040 \\
\hline 40.00 & 1.0738 & 1.0658 & 1.0575 & 1.0492 & 1.0407 & 1.0324 & 1.0240 & 1.0153 & 1.0067 \\
\hline 45.00 & 1.0757 & 1.0678 & 1.0595 & 1.0513 & 1.0429 & 1.0347 & 1.0264 & 1.0178 & 1.0093 \\
\hline 50.00 & 1.0775 & 1.0697 & 1.0616 & 1.0534 & 1.0451 & 1.0369 & 1.0288 & 1.0203 & 1.0119 \\
\hline 55.00 & 1.0794 & 1.0716 & 1.0635 & 1.0555 & 1.0471 & 1.0392 & 1.0311 & 1.0227 & 1.0144 \\
\hline 60.00 & 1.0811 & 1.0734 & 1.0653 & 1.0573 & 1.0491 & 1.0412 & 1.0334 & 1.0249 & 1.0169 \\
\hline
\end{tabular}


Table I. Continued

\begin{tabular}{|c|c|c|c|c|c|c|c|c|c|}
\hline \multirow{2}{*}{$\begin{array}{c}P \\
(M P a)\end{array}$} & \multicolumn{9}{|c|}{$T(K)$} \\
\hline & 278.15 & 288.15 & 298.15 & 308.15 & 318.15 & 328.15 & 338.15 & 348.15 & 358.15 \\
\hline & \multicolumn{9}{|c|}{$x=0.7012$} \\
\hline 0.10 & 1.0536 & 1.0451 & 1.0361 & 1.0273 & 1.0181 & 1.0089 & 0.9997 & 0.9901 & 0.9806 \\
\hline 1.00 & 1.0542 & 1.0455 & 1.0366 & 1.0278 & 1.0186 & 1.0094 & 1.0002 & 0.9907 & 0.9812 \\
\hline 5.00 & 1.0560 & 1.0474 & 1.0387 & 1.0299 & 1.0208 & 1.0117 & 1.0026 & 0.9932 & 0.9839 \\
\hline 10.00 & 1.0583 & 1.0498 & 1.0412 & 1.0324 & 1.0234 & 1.0145 & 1.0055 & 0.9964 & 0.9872 \\
\hline 15.00 & 1.0604 & 1.0521 & 1.0435 & 1.0349 & 1.0261 & 1.0173 & 1.0085 & 0.9994 & 0.9904 \\
\hline 20.00 & 1.0626 & 1.0543 & 1.0458 & 1.0373 & 1.0286 & 1.0199 & 1.0113 & 1.0023 & 0.9934 \\
\hline 25.00 & 1.0647 & 1.0564 & 1.0480 & 1.0397 & 1.0310 & 1.0225 & 1.0140 & 1.0051 & 0.9964 \\
\hline 30.00 & 1.0667 & 1.0586 & 1.0503 & 1.0420 & 1.0334 & 1.0250 & 1.0166 & 1.0079 & 0.9993 \\
\hline 35.00 & 1.0687 & 1.0607 & 1.0525 & 1.0442 & 1.0358 & 1.0275 & 1.0192 & 1.0106 & 1.0022 \\
\hline 40.00 & 1.0707 & 1.0628 & 1.0546 & 1.0465 & 1.0381 & 1.0299 & 1.0217 & 1.0133 & 1.0049 \\
\hline 45.00 & 1.0727 & 1.0648 & 1.0567 & 1.0486 & 1.0404 & 1.0323 & 1.0242 & 1.0158 & 1.0076 \\
\hline 50.00 & 1.0746 & 1.0668 & 1.0588 & 1.0508 & 1.0426 & 1.0345 & 1.0266 & 1.0183 & 1.0102 \\
\hline 55.00 & 1.0765 & 1.0687 & 1.0608 & 1.0529 & 1.0448 & 1.0368 & 1.0290 & 1.0208 & 1.0128 \\
\hline \multirow[t]{2}{*}{60.00} & 1.0782 & 1.0707 & 1.0627 & 1.0550 & 1.0468 & 1.0390 & 1.0311 & 1.0231 & 1.0152 \\
\hline & \multicolumn{9}{|c|}{$x=0.8028$} \\
\hline 0.10 & 1.0505 & 1.0418 & 1.0331 & 1.0242 & 1.0152 & 1.0061 & 0.9970 & 0.9876 & 0.9783 \\
\hline 1.00 & 1.0509 & 1.0423 & 1.0335 & 1.0247 & 1.0158 & 1.0066 & 0.9976 & 0.9882 & 0.9789 \\
\hline 5.00 & 1.0528 & 1.0443 & 1.0355 & 1.0268 & 1.0180 & 1.0090 & 1.0001 & 0.9908 & 0.9817 \\
\hline 10.00 & 1.0551 & 1.0467 & 1.0381 & 1.0295 & 1.0208 & 1.0119 & 1.0031 & 0.9940 & 0.9849 \\
\hline 15.00 & 1.0574 & 1.0490 & 1.0405 & 1.0320 & 1.0235 & 1.0147 & 1.0061 & 0.9971 & 0.9883 \\
\hline 20.00 & 1.0596 & 1.0513 & 1.0429 & 1.0345 & 1.0261 & 1.0174 & 1.0089 & 1.0001 & 0.9914 \\
\hline 25.00 & 1.0617 & 1.0535 & 1.0452 & 1.0369 & 1.0286 & 1.0201 & 1.0117 & 1.0030 & 0.9945 \\
\hline 30.00 & 1.0639 & 1.0558 & 1.0475 & 1.0393 & 1.0311 & 1.0227 & 1.0144 & 1.0059 & 0.9975 \\
\hline 35.00 & 1.0660 & 1.0579 & 1.0498 & 1.0416 & 1.0336 & 1.0252 & 1.0171 & 1.0086 & 1.0004 \\
\hline 40.00 & 1.0680 & 1.0600 & 1.0520 & 1.0439 & 1.0359 & 1.0277 & 1.0197 & 1.0113 & 1.0032 \\
\hline 45.00 & 1.0700 & 1.0621 & 1.0541 & 1.0462 & 1.0383 & 1.0301 & 1.0222 & 1.0140 & 1.0060 \\
\hline 50.00 & 1.0719 & 1.0642 & 1.0563 & 1.0484 & 1.0406 & 1.0325 & 1.0247 & 1.0165 & 1.0087 \\
\hline 55.00 & 1.0739 & 1.0662 & 1.0583 & 1.0505 & 1.0428 & 1.0348 & 1.0271 & 1.0190 & 1.0113 \\
\hline \multirow[t]{2}{*}{60.00} & 1.0757 & 1.0680 & 1.0602 & 1.0525 & 1.0448 & 1.0370 & 1.0293 & 1.0215 & 1.0138 \\
\hline & \multicolumn{9}{|c|}{$x=0.9039$} \\
\hline 0.10 & 1.0479 & 1.0391 & 1.0302 & 1.0214 & 1.0126 & 1.0036 & 0.9945 & 0.9853 & 0.9761 \\
\hline 1.00 & 1.0483 & 1.0395 & 1.0307 & 1.0219 & 1.0131 & 1.0041 & 0.9951 & 0.9859 & 0.9768 \\
\hline 5.00 & 1.0503 & 1.0415 & 1.0328 & 1.0241 & 1.0154 & 1.0065 & 0.9976 & 0.9886 & 0.9796 \\
\hline 10.00 & 1.0526 & 1.0440 & 1.0354 & 1.0268 & 1.0182 & 1.0095 & 1.0008 & 0.9919 & 0.9831 \\
\hline 15.00 & 1.0550 & 1.0464 & 1.0379 & 1.0294 & 1.0210 & 1.0123 & 1.0038 & 0.9950 & 0.9864 \\
\hline 20.00 & 1.0572 & 1.0488 & 1.0404 & 1.0320 & 1.0237 & 1.0151 & 1.0067 & 0.9980 & 0.9896 \\
\hline 25.00 & 1.0594 & 1.0510 & 1.0427 & 1.0344 & 1.0263 & 1.0178 & 1.0096 & 1.0011 & 0.9927 \\
\hline 30.00 & 1.0616 & 1.0533 & 1.0451 & 1.0369 & 1.0288 & 1.0205 & 1.0124 & 1.0040 & 0.9957 \\
\hline 35.00 & 1.0637 & 1.0555 & 1.0474 & 1.0393 & 1.0313 & 1.0231 & 1.0151 & 1.0068 & 0.9987 \\
\hline 40.00 & 1.0658 & 1.0577 & 1.0496 & 1.0416 & 1.0337 & 1.0256 & 1.0177 & 1.0096 & 1.0016 \\
\hline 45.00 & 1.0678 & 1.0598 & 1.0518 & 1.0439 & 1.0361 & 1.0281 & 1.0203 & 1.0122 & 1.0044 \\
\hline 50.00 & 1.0698 & 1.0619 & 1.0540 & 1.0462 & 1.0384 & 1.0306 & 1.0228 & 1.0149 & 1.0071 \\
\hline 55.00 & 1.0718 & 1.0640 & 1.0561 & 1.0484 & 1.0407 & 1.0329 & 1.0253 & 1.0175 & 1.0098 \\
\hline 60.00 & 1.0737 & 1.0659 & 1.0581 & 1.0505 & 1.0428 & 1.0351 & 1.0275 & 1.0199 & 1.0123 \\
\hline
\end{tabular}

